\title{
Variations in the Origins of Inferior Phrenic Arteries- An Evaluation with 256 Slice Multidetector Computed Tomography
}

\author{
Chinmay Bhimaji Kulkarni ${ }^{1}$ Sreekumar Karumathil Pullara ${ }^{1, \odot ~ N i r m a l ~ K u m a r ~ P r a b h u, ~}{ }^{1, \odot}$ \\ Minnie Pillay2,๑ Srikanth Moorthy ${ }^{1}$

\footnotetext{
${ }^{1}$ Department of Radiology, Amrita Institute of Medical Sciences, Amrita Vishwa Vidyapeetham, Cochin, Kerala, India

2Department of Anatomy, Amrita Institute of Medical Sciences, Amrita Vishwa Vidyapeetham, Cochin, Kerala, India
}

\begin{abstract}
Address for correspondence Chinmay Bhimaji Kulkarni, DNB, Department of Radiology, Amrita Institute of Medical Sciences and Research Centre, Amrita Lane, Ponekkra, Elamakkara P.O., Kochi, Kerala 682041, India (e-mail: drchinmaykulkarni@gmail.com,chidoc@rediffmail.com).
\end{abstract}

\begin{abstract}
Keywords

- arteries

- aorta

- multidetector computed tomography

- inferior phrenic artery

- celiac artery

- chemoembolization

- hemoptysis

Aim To evaluate the frequency and pattern of variations in the origins of right inferior phrenic artery (RIPA) and left inferior phrenic artery (LIPA) on 256 slice multidetector computed tomography (MDCT).

Materials and Methods MDCT abdominal images of 600 patients (male: 344, female: 256 ; mean age $56.45 \pm 12.96$ years) who underwent technically successful multiphase computed tomography were assessed for IPA variations with emphasis on their origins and results analyzed.

Results Both IPA origins were documented in all patients. Both RIPA and LIPA originated from the common trunk in 128 (21.3\%) patients. IPAs with common trunk most commonly originated from the aorta $(68,11.3 \%)$. Without common trunk RIPA most commonly originated from the aorta $(225,37.5 \%)$ and LIPA from the celiac artery $(278,46.3 \%)$. The least frequently detected IPA variations were RIPA originating from the common hepatic artery $(1,0.2 \%)$, superior mesenteric artery $(1,0.2 \%)$, and common truncus originating from left renal artery $(1,0.2 \%)$.

Conclusion MDCT demonstrates the IPA origins very well, enabling planning of interventional procedures related to IPA. Without common trunk RIPA most commonly originates from aorta and LIPA from the celiac artery. IPAs with common trunk most commonly originate from aorta.
\end{abstract}

\section{Introduction}

Inferior phrenic arteries (IPAs) are small caliber paired arteries supplying diaphragm with small branches to the liver, adrenal glands, and gastroesophageal junction. Though the right inferior phrenic artery (RIPA) and the left inferior phrenic artery (LIPA) commonly originate from the aorta or celiac artery, they frequently show variations in their origins. ${ }^{1}$ IPA is involved in various pathologic conditions like hemoptysis due to lower lobe lung abnormality, bleeding due to diaphragm/liver trauma, bleeding from gastroesophageal pathologies like Mallory-Weiss tear or tumor. ${ }^{2-6}$ The RIPA is the most common extrahepatic blood supply to hepatocellular carcinoma (HCC), especially those located near the bare area of the liver and exophytic lesions abutting the undersurface of the diaphragm. ${ }^{7}$ Knowledge of the variations helps to
DOI https://doi.org/

10.1055/s-0040-1710166

ISSN 2457-0214.
License terms

(1) (1) $\odot \circledast$ 
achieve effective treatment of HCC by transarterial chemoembolization (TACE) and other pathologies related to IPA. We undertook this study to document the frequency and pattern of variations in the origins of IPA.

\section{Aim}

The study aims to evaluate the frequency and pattern of variations in the origins of RIPA and LIPA on 256 slice multidetector computed tomography (MDCT).

\section{Materials and Methods}

\section{Study Population}

An institutional review board approved this retrospective study. Informed consent was taken before the CT scans. Between January 2018 and November 2019, abdominal CT angiography (CTA) images of 600 patients (male:female = 344:256; mean age $56.45 \pm 12.96$ years) who underwent technically successful multiphase CT for cirrhosis without HCC or hepatic vascular disease, mesenteric ischemia, gastrointestinal bleed, peripheral arterial disease, donor patients for renal/liver transplantation, renal artery stenosis, and aortic aneurysms were included. None of the patients included in the study had primary or metastatic hepatic neoplasm, pleural or diaphragmatic disease, at the time of the CT scan. Images of patients who had severe atherosclerosis, and/or movement artifacts preventing assessment of the IPAs were also excluded from the study ( - Fig. 1).

\section{CT Technique}

Multiphase computed tomography was performed with a 256-section multidetector CT scanner (Philips 256 slice ICT, Philips Healthcare). Scanning parameters are shown in

- Table 1. Contrast-enhanced images were obtained in the bolus-triggered arterial phase and delayed venous phases. A region of interest was placed over the descending thoracic aorta, $2 \mathrm{~cm}$ proximal to the diaphragm, and the scanning was initiated 6 seconds after the threshold of $150 \mathrm{HU}$ was reached. Patients were scanned at $120 \mathrm{kV}$ with an active dose reduction index (DRI-20).

\section{Image Interpretation}

The image analysis was done on dedicated workstations (Philips Healthcare) which allowed three-dimensional postprocessing with multiplanar image reformatting and maximum intensity projections. Arterial phase CT images

Table 1 Multidetector computed tomography parameters used for scanning patients

\begin{tabular}{|c|c|}
\hline Parameter & Comment \\
\hline Peak voltage (kVp) & 120 kVP \\
\hline Effective (mAs) & 150-350 mAs (DRI-20) \\
\hline Rotation time (s) & $0.5 \mathrm{~s}$ \\
\hline Detector collimation & $128 \times 0.625 \mathrm{~mm}$ \\
\hline Pitch & 0.914 \\
\hline Matrix & $512 \times 512$ \\
\hline $\begin{array}{l}\text { Reconstruction slice } \\
\text { thickness }\end{array}$ & $1.0 \mathrm{~mm}$ \\
\hline $\begin{array}{l}\text { Reconstruction } \\
\text { method }\end{array}$ & iDose-4 \\
\hline IV contrast & $\begin{array}{l}\text { Omnipaque } 350 \text { (iohexol); volume: } \\
1.5 \mathrm{~mL} / \mathrm{kg} \text {; rate: } 4-6 \mathrm{~mL} / \mathrm{s} \text {. }\end{array}$ \\
\hline Scan delay & $\begin{array}{l}\text { Hepatic AP: } 15 \text { s; other AP: } 6 \text { s (bolus } \\
\text { tracking; ROI-descending aorta, } 2 \mathrm{~cm} \\
\text { above). }\end{array}$ \\
\hline
\end{tabular}

Abbreviations: AP, arterial phase; DRI, dose reduction index; HU, Hounsfield; IV, intravenous; ROI, region of interest.

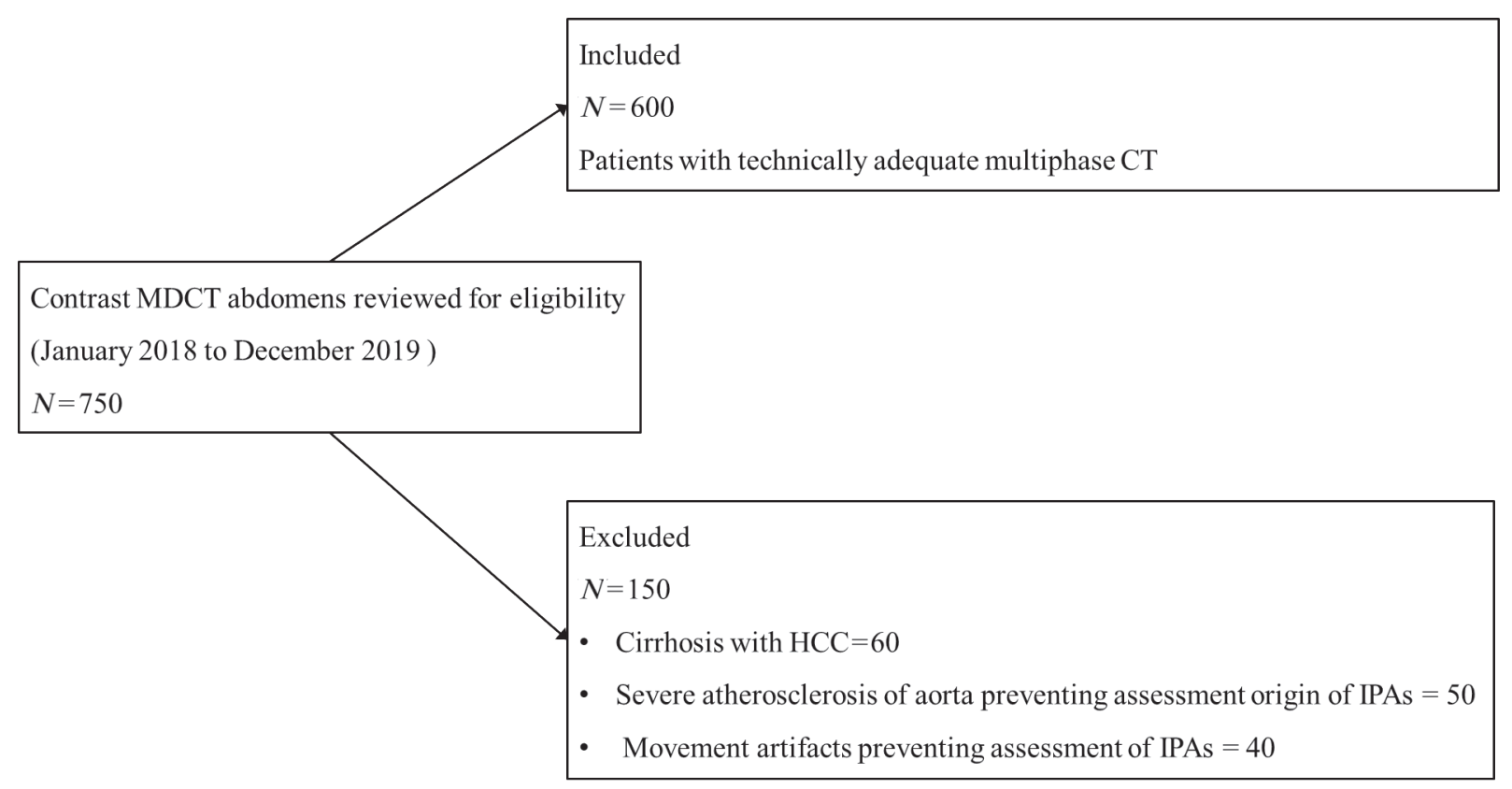

Fig. 1 Diagram showing included and excluded patients in the study. CT, computed tomography; HCC, hepatocellular carcinoma; IPA, inferior phrenic artery; MDCT, multidetector computed tomography. 
with 1-mm slice thickness were used to assess the IPA. All images were reviewed by a radiologist with 10 years of experience in abdominal CT imaging. The IPAs were assessed with emphasis on their origins and results analyzed.

\section{Statistical Analysis}

Statistical analysis was performed using IBM SPSS Statistics 20.0 software (IBM Corp.). The data was analyzed using descriptive statistical methods. Quantitative variables were expressed as mean values and standard deviations.

\section{Results}

Both IPA origins were documented in all patients. IPAs were divided into two groups: those originating from a common trunk and those originating independently from different sources without a truncus. Both RIPA and LIPA originated from a common trunk in 128 (21.3\%) patients ( - Fig. 2). The truncus originated from the aorta in $68(11.3 \%)$ patients and from the celiac artery in $58(9.6 \%)$ patients ( - Fig. 3). Both RIPA and LIPA originated from a common trunk in one
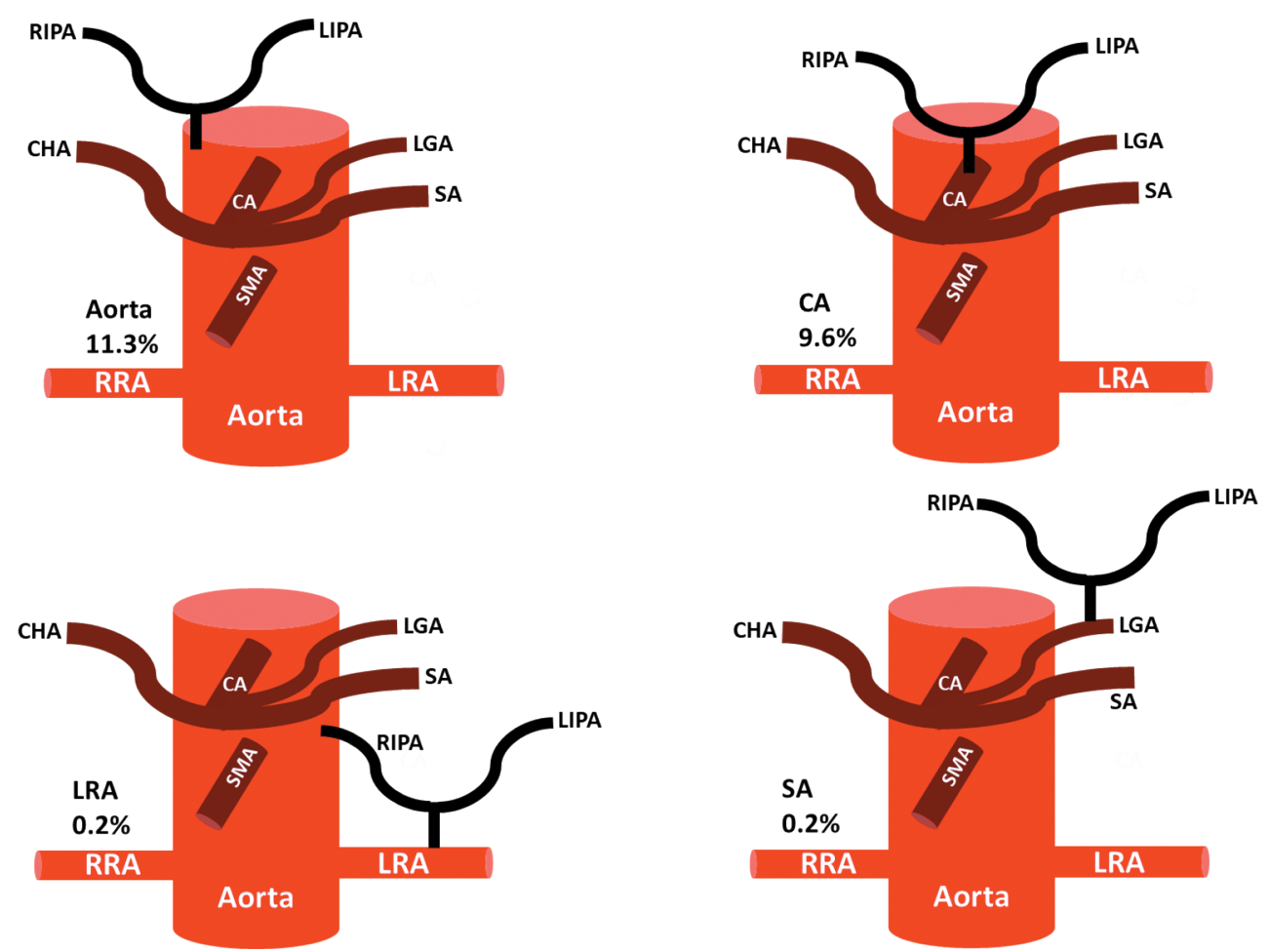

Fig. 2 Schematic representation of origin of inferior phrenic arteries with common trunk. CA, celiac artery, CHA, common hepatic artery; LGA, left gastric artery; LIPA, left inferior phrenic artery; LRA, left renal artery; RIPA, right inferior phrenic artery; RRA, right renal artery; SA, splenic artery; SMA, superior mesenteric artery.

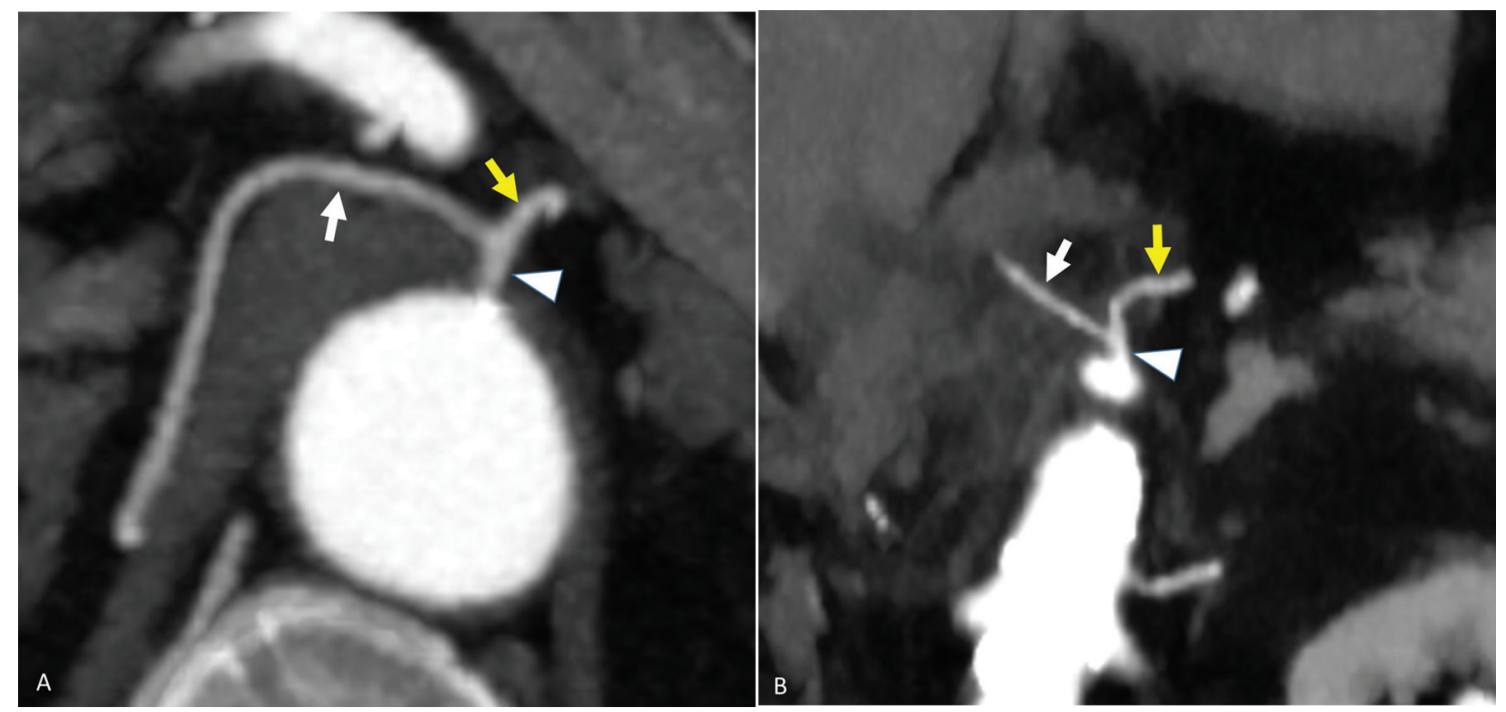

Fig. 3 Multidetector computed tomography arterial phase maximum intensity projection images show right IPA (A, B white arrow) and left IPA (A, B yellow arrow) originating from aorta (A arrowhead) and celiac artery (B arrowhead) as common trunk. IPA, inferior phrenic artery. 
patient $(0.2 \%)$ each from the left renal artery and left gastric artery (-Figs. 4 and $\mathbf{5}$ ). The RIPA and the LIPA originated independently in 472 (78.6\%) patients (-Figs. 6 and 7). The RIPA most commonly originated from the abdominal aorta in 225 (37.5\%) patients, followed by the celiac artery in 177 (29.5\%), right renal artery in 59 (9.8\%), left gastric artery in $9(1.5 \%)$, and $1(0.2 \%)$ each from common hepatic artery (CHA) and superior mesenteric artery (SMA), respectively (-Figs. 8-11). The LIPA most commonly originated from the celiac artery in 278 (46.3\%) followed by the abdominal aorta in 181 (30.1\%), left renal artery in 7 (1.1\%), and splenic artery in $6(1.0 \%)$ (-Figs. 8-10, 12, 13). In patients with IPA originating from the aorta, the origins were from the anterior, lateral. or posterior aspect of the aorta. The least frequently detected IPA variations were RIPA originating from the CHA and SMA. We also observed that in patients with multiple renal arteries, IPA originated from the uppermost renal artery (-Fig. 11).

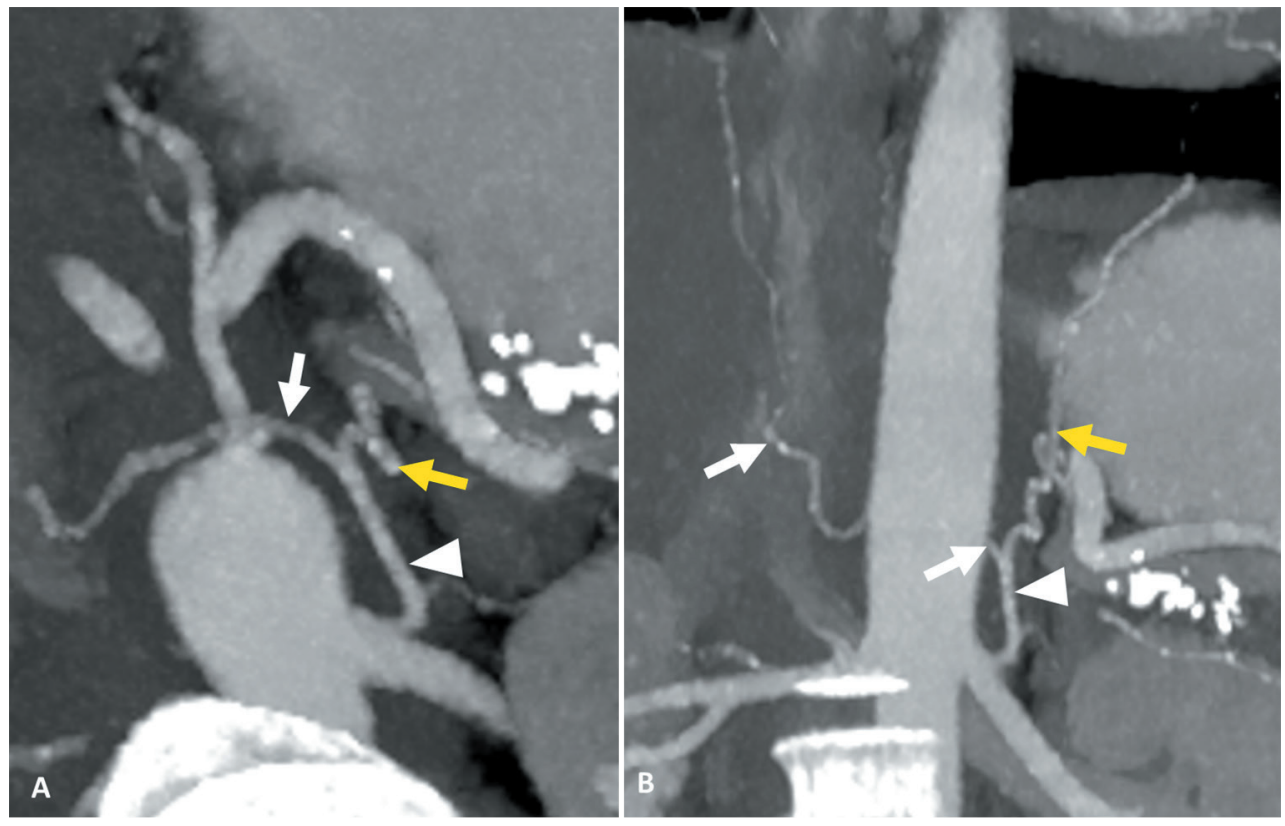

Fig. 4 Multidetector computed tomography arterial phase maximum intensity projection images show right IPA (A, B white arrow) and left IPA (A, B yellow arrow) originating from left renal artery (A, B arrowhead) as common trunk. IPA, inferior phrenic artery.
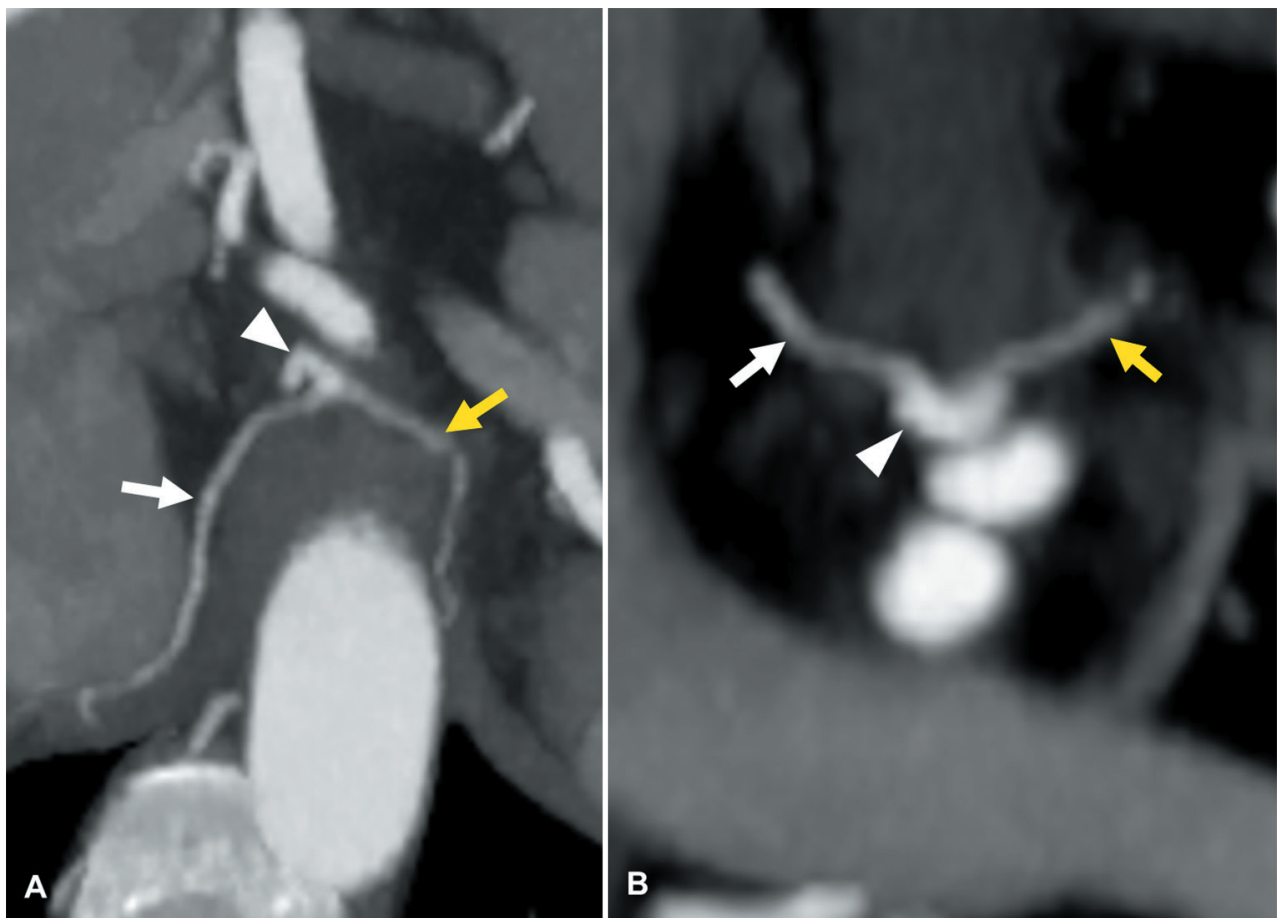

Fig. 5 Multidetector computed tomography arterial phase maximum intensity projection images show right IPA (A, B white arrow) and left IPA (A, B yellow arrow) originating from left gastric artery (A, B arrowhead) as common trunk. IPA, inferior phrenic artery. 

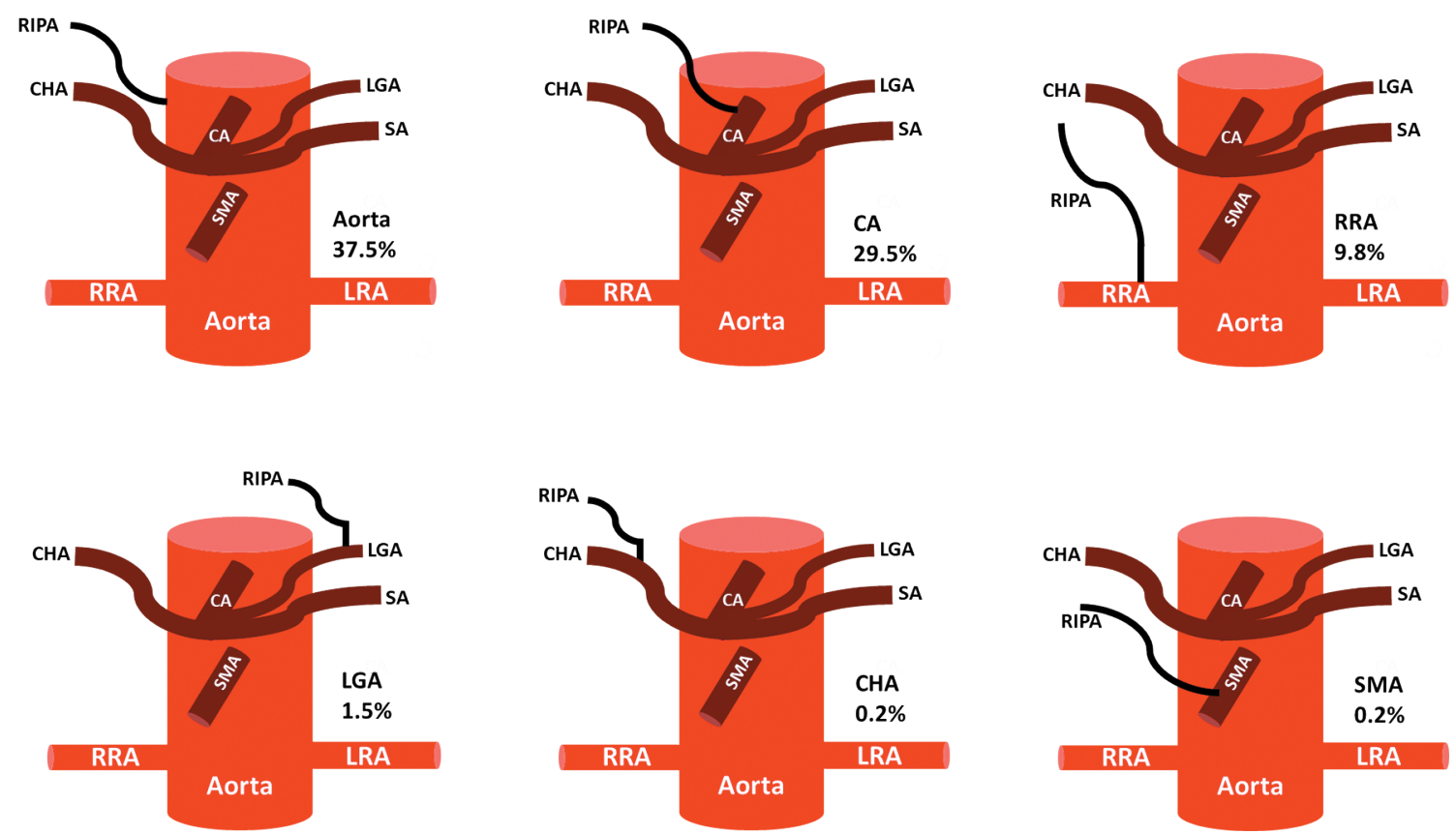

Fig. 6 Schematic representation of origin of RIPAs from different sources without common trunk. CA, celiac artery; CHA, common hepatic artery; LGA, left gastric artery; LRA, left renal artery; RIPA, right inferior phrenic artery; RRA, right renal artery; SA, splenic artery; SMA, superior mesenteric artery.
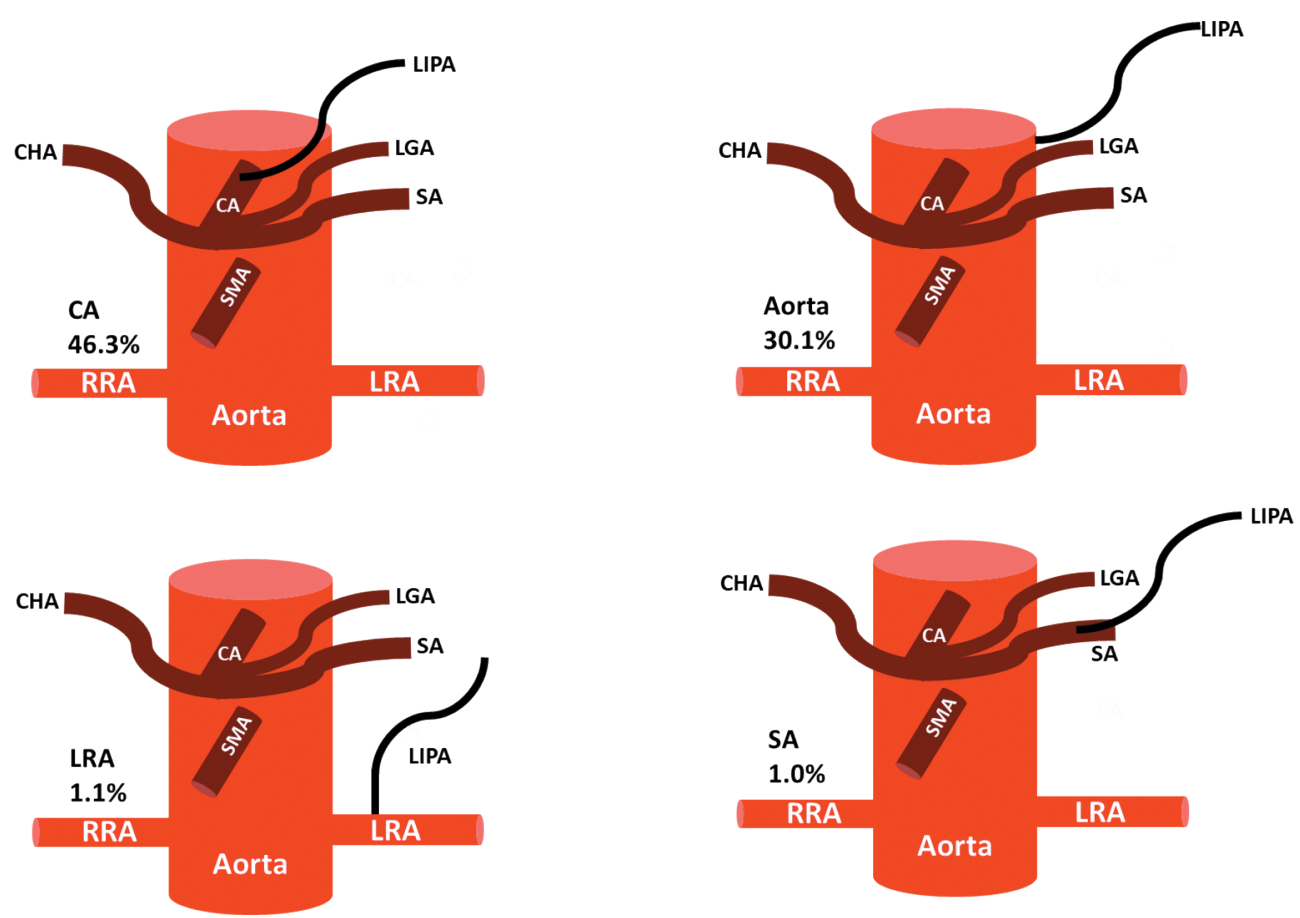

Fig. 7 Schematic representation of origin of LIPAs from different sources without common trunk. CA, celiac artery; CHA, common hepatic artery; LGA, left gastric artery; LIPA, left inferior phrenic artery; LRA, left renal artery; RRA, right renal artery; SA, splenic artery; SMA, superior mesenteric artery.

\section{Discussion}

Advances in CT technology have expanded its role in vascular imaging. Faster scanning and multiplanar reconstruction capabilities allow clear visualization of smaller arteries in different parts of the body. This, in turn, helps in studying vascular anatomy, variations, anomalies, and various vascular pathologies. Though digital subtraction angiography (DSA) is the gold standard for vascular imaging, because of wide variations associated with the origins of the small abdominal visceral arteries, overlapping structures during DSA, poor breath-hold of the patients, and bowel gas artifacts, it is not always easy to detect the arterial anatomy/pathology on DSA. MDCT with three-dimensional reconstruction capability 


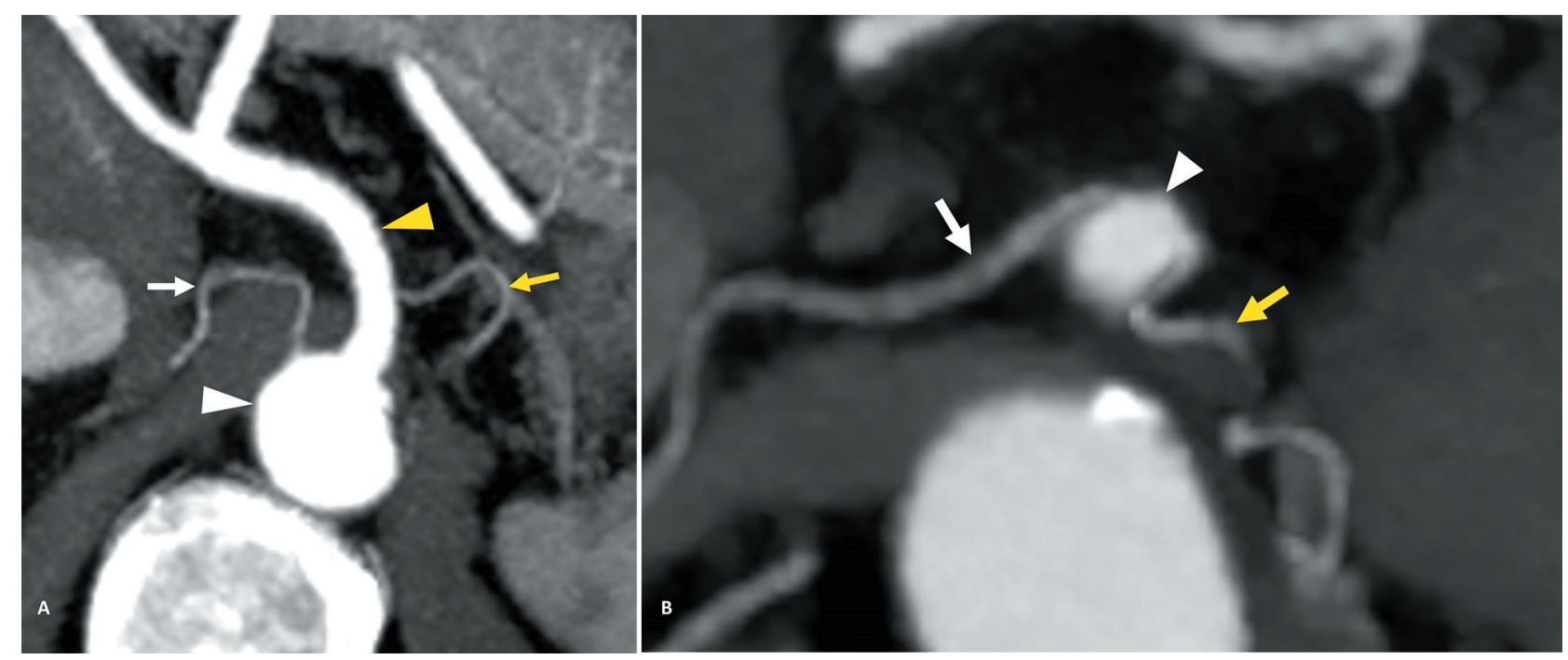

Fig. 8 (A, B) Multidetector computed tomography arterial phase maximum intensity projection images show right inferior phrenic artery (RIPA, white arrow) and left inferior phrenic artery (LIPA, yellow arrow) originating separately without common truncus. (A) RIPA originates from aorta (white arrowhead) and LIPA from celiac artery (yellow arrowhead). (B) Both RIPA and LIPA originate from celiac artery (white arrowhead).
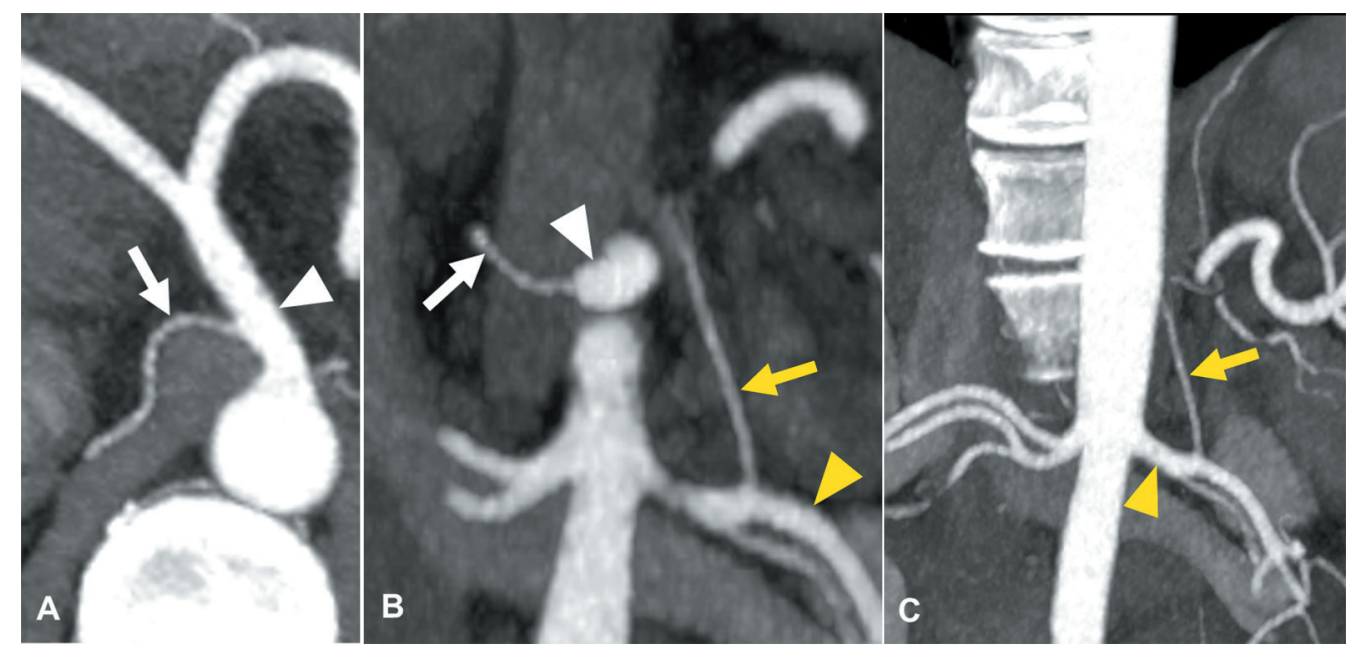

Fig. 9 Multidetector computed tomography arterial phase maximum intensity projection images show right IPA (A, B white arrow) originating from the celiac artery (A, B arrowhead) and left IPA (B, C yellow arrow) originating from left renal artery (B, C yellow arrowhead). IPA, inferior phrenic artery.

helps in delineating the anatomy and detecting the pathology accurately. ${ }^{1,8}$ CTA prior to the vascular interventional procedure helps in identifying the culprit artery and its variations allowing us to draw up a clear strategy and plan the embolization technique to be used. These in turn significantly reduce the procedure time, contrast usage, and radiation exposure. IPA are millimetric sized arteries and not always easy to visualize. The appropriately timed arterial phase is required for the adequate visualization of the IPA. We had obtained contrast-enhanced images using bolus triggering technique which helps in adequate opacification of arteries. Studies have shown that a higher rate of injection of the contrast improves the detection in gastrointestinal bleed patients which indirectly means better visualization of the smaller arteries like IPA. ${ }^{9}$ Technically inadequate CT scans and those with movement artifacts pose a challenge in the assessment of the IPA origins. Severe atherosclerotic calcification of the aorta or occlusion of the aorta and celiac artery also makes the interpretation of the origins of IPA difficult.,10 Ozbulbul et al in their study of 200 patients had shown the importance of arterial-phase images for demonstrating the origins of IPAs in a population without liver disease..$^{10}$ In our study, we had a heterogeneous patient population including cirrhotic patients without HCC (33.3\%). We were able to document the origins of both the RIPA and LIPA in all patients.

\section{Variations in the Origins of IPA and the Embryological Basis}

The origin of IPAs can be from the aorta, celiac artery, or from other visceral arteries. ${ }^{1,11}$ In the study conducted by Basile et al the authors reported 13 different variations in the origin of IPA using MDCT. However, this study did not separately mention the origin from a common trunk. ${ }^{12}$ Loukas et al conducted a study on cadavers and classified the origins of LIPA and RIPA 


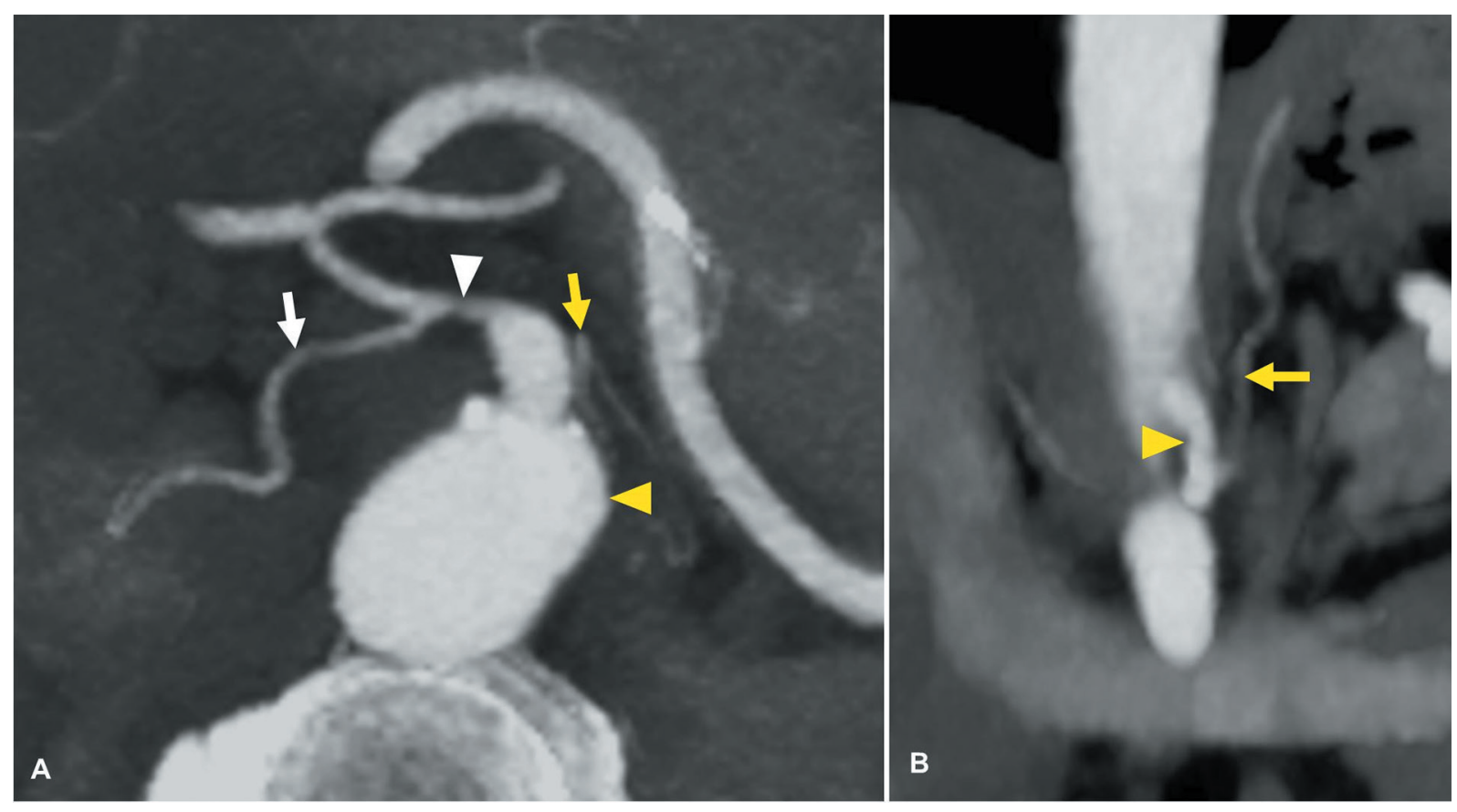

Fig. 10 (A, B) Multidetector computed tomography arterial phase maximum intensity projection images show right inferior phrenic artery (RIPA, white arrow) and left inferior phrenic artery (LIPA, yellow arrow) originating separately without common truncus. (A) RIPA originates from left gastric artery (white arrowhead) and LIPA from aorta (yellow arrowhead). (B) LIPA originates from left gastric artery (yellow arrowhead).
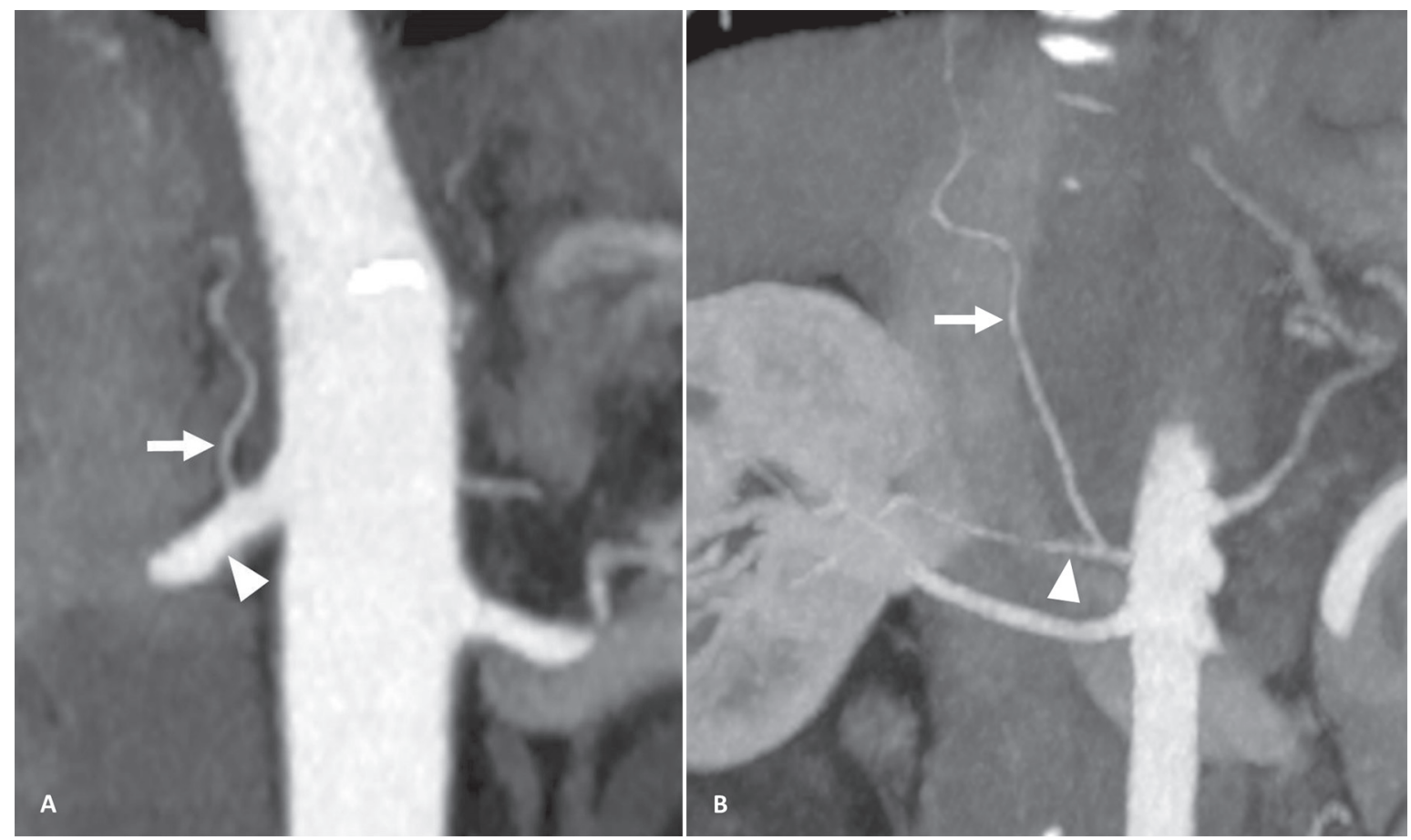

Fig. 11 Multidetector computed tomography arterial phase maximum intensity projection images show right inferior phrenic artery (RIPA; A, B white arrow) originating separately without common truncus. (A) RIPA originates from right renal artery (white arrowhead); (B) RIPA originates from accessory superior right renal artery (white arrowhead).

into five groups. ${ }^{11}$ In both these studies, the most common site of origin of IPA was the celiac artery. Ozbulbul et al reported the variations in IPA origin in 200 patients without liver disease on CTA. ${ }^{10}$ The authors have reported the rare origin of RIPA from the proper hepatic artery. Aslaner et al also studied the variations in the IPA origins on CTA in 1,000 patients. ${ }^{13}$ The authors reported common trunk of IPA origin in 295 (29.5\%) patients, with common trunk originating in most of the cases from the aorta (16.4\%) followed by celiac artery (12.6\%), right renal artery $(0.4 \%)$, and left gastric artery $(0.1 \%)$, in descending order. Our study is one of the larger studies in the printed literature in terms of the number of patients analyzed for IPA origin variations. We found 12 different types of variations in the origins of RIPA and LIPA (- Table 2). In our study, both 
RIPA and LIPA originated from a common trunk in 128 (21.3\%) patients. As in the previous study, common trunk originated from the aorta in most patients $(128,21.3 \%)$, followed by the celiac artery in $58(9.6 \%)$ patients. We also found the rare origins of the common trunk from the left gastric artery $(1,0.2 \%)$ and left renal artery $(1,0.2 \%)$ in our patients (-Figs. 4 and 5). In fact, the common truncus from the left renal artery has not been reported in the previous studies (-Fig. 4). However, we did not observe a rare common trunk originating from the right renal artery as reported by the previous authors. ${ }^{13}$ The RIPA and the LIPA originated independently in 472 (78.6\%) patients. In patients without a common trunk, RIPA most commonly originated from the aorta $(225,37.5 \%)$ and LIPA from a celiac artery $(278,46.3 \%)$. This contrasts with the previous studies which have observed that RIPA most commonly originates from the celiac artery. ${ }^{11,12}$ We observed that $46.3 \%$ of LIPA originated from the celiac artery. This observation is similar to other studies. ${ }^{12,13}$ We discovered a RIPA originating from SMA (0.2\%) and LIPA originating from the splenic artery (1.0\%), which have not been described in prior studies. As documented previously, in our study also, most of the IPAs originated from a segment of the aorta between the diaphragm and the renal arteries consistently. ${ }^{13}$ However, in one patient, the LIPA originated from the left lateral wall from the lower thoracic aorta just above the level of diaphragm (-Fig. 12).

It is essential to understand the embryological development of IPA to appreciate the anatomical logic behind the variations of IPA origin. Various theories have been proposed to explain the variations in IPA origin. Three groups of branches arise from the primitive dorsal aortae: the ventral, dorsal, and dorsolateral groups. Most of the primitive branches disappear and the ventral splanchnic arteries persist as a celiac artery, SMA, and inferior mesenteric artery to supply the foregut, midgut, and hindgut structures, respectively. The lateral splanchnic arteries persist as renal, suprarenal, phrenic, and gonadal arteries and supply the structures from intermediate mesoderm. The dorsolateral group of arteries persists as intercostal and lumbar arteries. According to "Felix's ladder theory," IPAs are said to be formed by the cranial group of lateral splanchnic arteries called "Rete arteriosus Urogenitale," which can explain the variations in origin between other lateral branches like renal and gonadal arteries. ${ }^{14}$ However, this theory does not explain IPA origin from celiac artery and a common trunk of origin from the abdominal aorta which is observed in majority of the patients. During fetal development, most primitive arteries from the ventral, lateral, and dorsal group disappear. The persistence of longitudinal channels between these ventral, lateral, and dorsal primitive vessels could explain the fact that IPAs can originate from the celiac artery and aorta with common truncus. ${ }^{15}$ According to another theory proposed by Isogai et al, the adrenal primordium (cranial half of paraaortic ridge) is supplied by few branches from the gonadal artery and/or abdominal aorta, during day 14 of embryonic life. The most cranial part of these adrenal arteries, which are the prospective IPAs, establishes their definitive branching pattern of the adult by day $15 .{ }^{16}$ This theory provides an explanation for the celiac origin of IPA as well as its origin as a common trunk from the aorta.

Knowing the origins of IPA is useful in various clinical scenarios. TACE is an important treatment option for unresectable HCC. It improves the prognosis of the patient by local tumor control and serves as a bridging procedure prior to a liver transplant. ${ }^{17}$ HCCs are mainly supplied by hepatic arterial branches. However, depending on the size and location, it can recruit extrahepatic collateral blood supply. Identifying these collateral arteries and targeting them is important to achieve complete embolization. RIPA is the most common extrahepatic artery supplying the HCCs, especially the ones located in the peripheral posterior segments of the right lobe and near the bare area of the liver.7 Prior to TACE, a detailed analysis of the arterial supply to the tumor is needed to achieve effective embolization. Visualization of the distal ascending portion of the IPA near the liver surface on CT may be an indicator of the extrahepatic supply of HCC by IPA. ${ }^{18}$ This warrants an IPA angiogram during TACE. IPAs are involved in transpleural systemic-pulmonary artery anastomosis. In inflammatory lower lung pathologies,
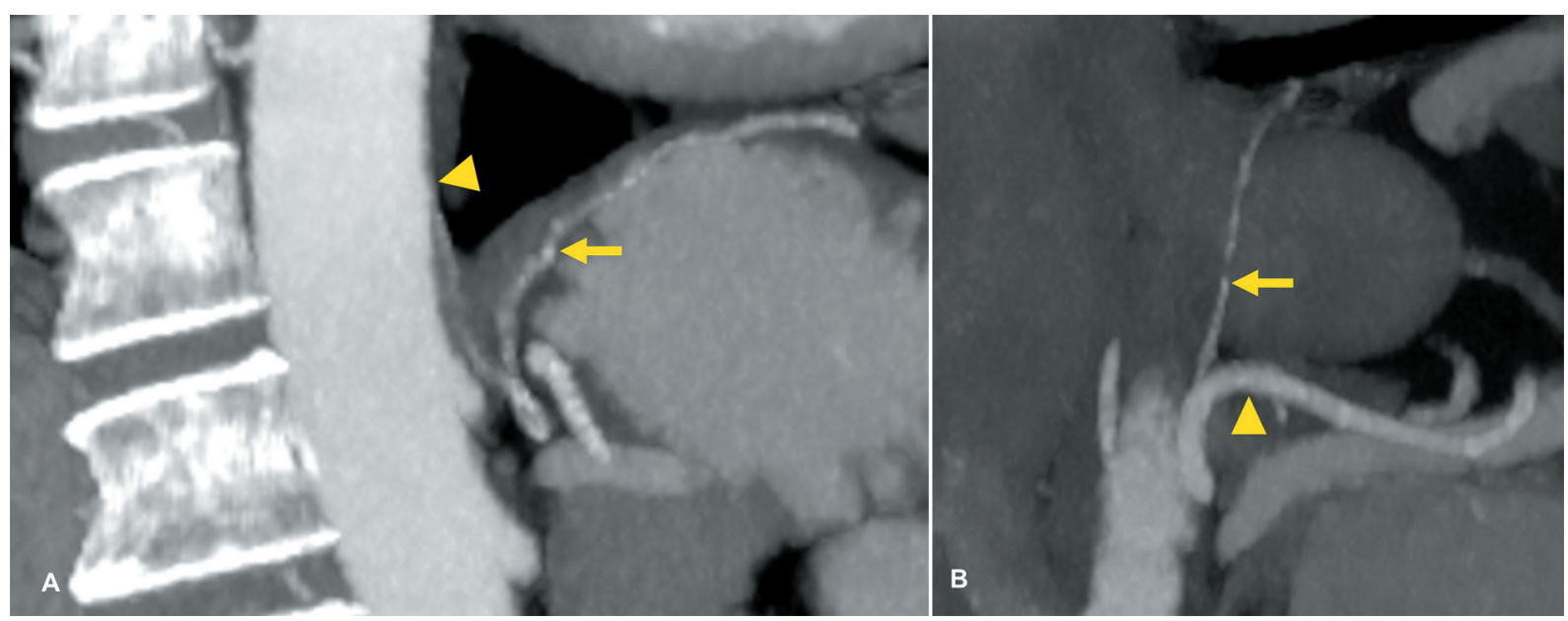

Fig. 12 Multidetector computed tomography arterial phase maximum intensity projection images show left inferior phrenic artery (LIPA; A, B yellow arrow) originating separately without common truncus. (A) LIPA originates from thoracic aorta (yellow arrowhead). (B) LIPA originates from splenic artery (yellow arrowhead). 

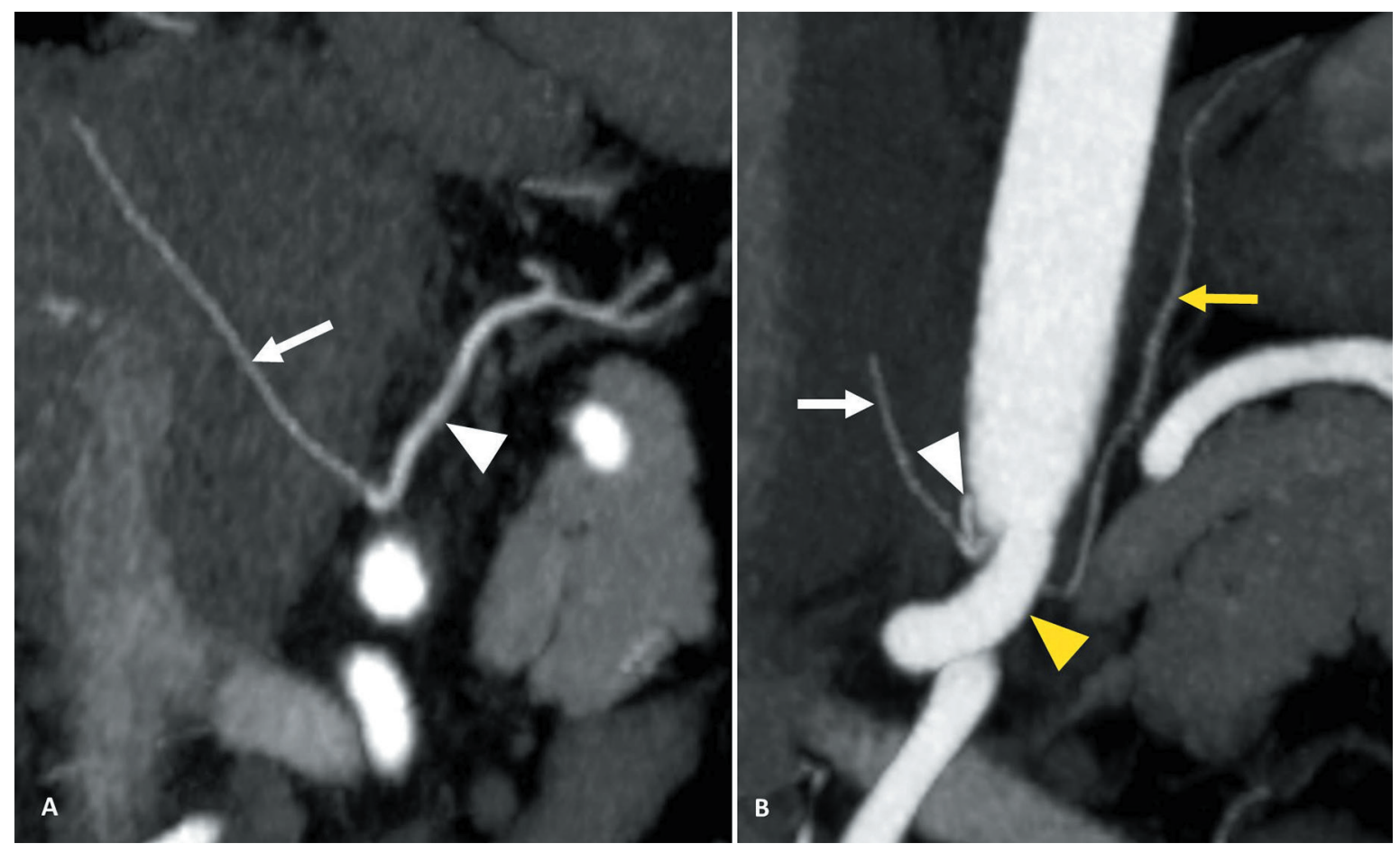

Fig. 13 Multidetector computed tomography arterial phase maximum intensity projection images show right IPA (A, B white arrow) originating from the left gastric artery (A,B white arrowhead) and left IPA (B, yellow arrow) originating from celiac artery (B yellow arrowhead). IPA, inferior phrenic artery.

Table 2 Variations in the origins of RIPA and LIPA

\begin{tabular}{|l|l|l|}
\hline \multicolumn{2}{|l|}{$N=600$} & \multicolumn{1}{l|}{ RIPA+LIPA } \\
\hline Common origin & \multicolumn{1}{l|}{$68(11.3 \%)$} \\
\hline Aorta & \multicolumn{1}{l|}{$58(9.6 \%)$} \\
\hline Celiac & $1(0.2 \%)$ \\
\hline Left gastric artery & $1(0.2 \%)$ & LIPA \\
\hline Left renal artery & RIPA & $181(30.1 \%)$ \\
\hline Separate origin & $225(37.5 \%)$ & $278(46.3 \%)$ \\
\hline Aorta & $177(29.5 \%)$ & 0 \\
\hline Celiac artery & $59(9.8 \%)$ & $7(1.1 \%)$ \\
\hline Right renal artery & 0 & 0 \\
\hline Left renal artery & $9(1.5 \%)$ & 0 \\
\hline Left gastric artery & $1(0.2 \%)$ & $6(1.0 \%)$ \\
\hline Common hepatic artery & 0 & 0 \\
\hline Splenic artery & $1(0.2 \%)$ & 0 \\
\hline SMA &
\end{tabular}

Abbreviations: LIPA, left inferior phrenic artery; RIPA, right inferior phrenic artery; SMA, superior mesenteric artery.

there is a compensatory increase in the systemic circulation, which may result in rupture of these systemic communications and hemoptysis. ${ }^{2,3,19}$ Occasionally, IPAs might be the only source of hemoptysis, especially in lower lobe lung pathologies. Hence, locating the IPA and treating it becomes critical for patient management. IPA may be the source of bleeding secondary to diaphragmatic or hepatic trauma or post liver transplant procedure due to inadequate ligation., ${ }^{4,20}$ LIPA is involved in anastomosis with the left anterior descending coronary artery, which may be of importance in patients with atherosclerotic ischemic heart diseases. ${ }^{21}$ LIPA supplies gastroesophageal junction and could be a source of bleed in post-gastrectomy patients (especially if IPA arises from the LGA).,5,622 LIPA is involved in the anastomosis with pericardiophrenic arteries and may be a source of traumatic pericardial tamponade, which can be managed effectively by LIPA embolization. ${ }^{21}$

Having a thorough knowledge of the vascular anatomy and being familiar with variations of IPA help to bail out the interventional radiologists from difficult situations faced on day-to-day basis. IPA should be an inherent part of the checklist while reporting or doing an interventional procedure in that region.

Our study had a few limitations. First is the retrospective nature of the study and second is the lack of confirmation of the variations detected on CTA images with DSA. We did not obtain DSA images of the patients as it was not indicated.

\section{Conclusion}

MDCT demonstrates the IPA origins very well, enabling planning of interventional procedures related to IPA. Without common trunk, RIPA most commonly originates from aorta and LIPA from the celiac artery. IPAs with common trunk 
most commonly originate from aorta. Being familiar with the variations of IPA origins is of great clinical relevance.

\section{Conflict of Interest}

The authors declare that they have no conflict of interest.

\section{Acknowledgment}

We would like to acknowledge the invaluable advice and support of our colleagues in the Departments of Anatomy and Bio-Statistics, Amrita Institute of Medical Sciences. This work would not have been possible without the dedication and skill of our radiology technicians and nursing staff.

\section{References}

1 Gwon DI, Ko GY, Yoon HK, et al. Inferior phrenic artery: anatomy, variations, pathologic conditions, and interventional management. Radiographics 2007;27(3):687-705

2 Webb WR, Jacobs RP. Transpleural abdominal systemic artery-pulmonary artery anastomosis in patients with chronic pulmonary infection. AJR Am J Roentgenol 1977; 129(2):233-236

3 Chun HJ, Byun JY, Yoo SS, Choi BG. Added benefit of thoracic aortography after transarterial embolization in patients with hemoptysis. AJR Am J Roentgenol 2003;180(6):1577-1581

4 Mizobata Y, Yokota J, Yajima Y, Sakashita K. Two cases of blunt hepatic injury with active bleeding from the right inferior phrenic artery. J Trauma 2000;48(6):1153-1155

5 Carsen GM, Casarella WJ, Spiegel RM. Transcatheter embolization for treatment of Mallory-Weiss tears of the esophagogastric junction. Radiology 1978;128(2):309-313

6 Smith DC, Kitching GB. Angiographic demonstration of esophagogastric bleeding from the inferior phrenic artery. Radiology 1977;125(3):613-614

7 Gokan T, Hashimoto T, Matsui S, Kushihashi T, Nobusawa $\mathrm{H}$, Munechika H. Helical CT demonstration of dilated right inferior phrenic arteries as extrahepatic collateral arteries of hepatocellular carcinomas. J Comput Assist Tomogr 2001;25(1):68-73

8 Lee AJ, Gomes AS, Liu DM, Kee ST, Loh CT, McWilliams JP. The road less traveled: importance of the lesser branches of the celiac axis in liver embolotherapy. Radiographics 2012;32(4):1121-1132

9 Kulkarni C, Moorthy S, Sreekumar K, et al. In the workup of patients with obscure gastrointestinal bleed, does 64-slice MDCT have a role? Indian J Radiol Imaging 2012;22(1):47-53
10 Ozbulbul NI, Yurdakul M, Tola M, Akdogan G, Olcer T. Can multidetector row CT visualize the right and left inferior phrenic artery in a population without disease of the liver? Surg Radiol Anat 2009;31(9):681-685

11 Loukas M, Hullett J, Wagner T. Clinical anatomy of the inferior phrenic artery. Clin Anat 2005;18(5):357-365

12 Basile A, Tsetis D, Montineri A, et al. MDCT anatomic assessment of right inferior phrenic artery origin related to potential supply to hepatocellular carcinoma and its embolization. Cardiovasc Intervent Radiol 2008;31(2):349-358

13 Aslaner R, Pekcevik Y, Sahin H, Toka O. Variations in the origin of inferior phrenic arteries and their relationship to celiac axis variations on CT Angiography. Korean J Radiol 2017;18(2):336-344

14 Felix W. Development of urogenital organs. In: Keibel F, Mall FP, eds. The Manual of Human Embryology. 2nd ed. Philadelphia, PA: J.B. Lippincott Company; 191219

15 Gürses İA, Gayretli Ö, Kale A, Öztürk A, Usta A, Şahinoğlu K. Inferior phrenic arteries and their branches, their anatomy and possible clinical importance: an experimental cadaver study. Balkan Med J 2015;32(2):189-195

16 Isogai S, Horiguchi M, Hitomi J. The para-aortic ridge plays a key role in the formation of the renal, adrenal and gonadal vascular systems. J Anat 2010;216(6):656-670

17 Gonsalves CF, Brown DB. Chemoembolization of hepatic malignancy. Abdom Imaging 2009;34(5):557-565

18 Kim HC, Chung JW, An S, Son KR, Jae HJ, Park JH. Hepatocellular carcinoma: detection of blood supply from the right inferior phrenic artery by the use of multi-detector row CT. J Vasc Interv Radiol 2008;19(11):1551-1557

19 Lee DH, Hwang JC, Lim SM, Yoon HK, Sung KB, Song HY. Pleural and pulmonary staining at inferior phrenic arteriography mimicking a tumor staining of hepatocellular carcinoma. Cardiovasc Intervent Radiol 2000;23(2):109-113

20 Hong SS, Kim AY, Kim HJ, et al. Inferior phrenic arterial bleeding after adult liver transplantation: incidence, clinical manifestations, and predictive CT features. AJR Am J Roentgenol 2006;187(1):W15-9

21 Jones BV, Vu D. Diagnosis of posttraumatic pericardial tamponade by plain film and computed tomography and control of bleeding by embolotherapy of the left inferior phrenic artery. Cardiovasc Intervent Radiol 1993;16(3):183-185

22 Zeng R, Yao Z, Chen Y, Xu Z, Chen Y, Liu J. Variant arterial supply to the lesser curvature of the stomach and duodenum from double inferior phrenic arteries. Surg Radiol Anat 2015;37(7):867-869 